\title{
INTERKULTURELLES UND INTERTEXTUELLES IN GEBRAUCHSTEXTEN
}

A. RENAULT TWINGO. DER MACHT DIE WELT VERRÜCKT.

APPARTEMENT, CA. $6 \mathrm{M}^{2}$, SCHLAFZ., GÄSTEZ., GR. ABSTELLRAUM, WARM, MÖBLIERT UND AB SOFORT.

EIN ZIMMER MIT AUSSICHT UND DIE LAGE NACH WUNSCH. FÜR TWINGO KEIN PROBLEM. DAZU GIBT'S SEIN UNSCHLAGBARES RAUMANGEBOT OHNE MAKLER. (freundin 7/97/S. 151)

Wenn man sich die Werbung für den PKW Renault Twingo anschaut, sieht man, daß das Ganze eigentlich metaphorisch gedacht ist. Es handelt sich offenbar um eine komplexe Metaphorik, die nicht nur auf ein Lexem beschränkt ist, sondern fast über den ganzen Text hin entfaltet ist, jedoch nicht konsequent bis Ende durchgehalten wird.

Ähnliches können wir auch im Text "Stellensuche: Vorsprung für Internet-Surfer" (freundin 19/96/S.126) feststellen, wo wir u. a. folgendes lesen können: "Wer sich online nach einem Job umsieht, ist Mitbewerbern einen SCHRITT voraus: Meist ist der Weg zum neuen Job eher ein HOLPRIGER PFAD als eine Autobahn: ENGPAB auf dem Arbeitsamt - interessante Angebote sind Mangelware. Mehr Auswahl bieten die Anzeigenmärkte der Tageszeitungen. Doch da antworten Hunderte - AKUTE STAUGEFAHR für Ihre Bewerbung. Die SCHNELLSTE ROUTE führt über den Daten-HIGHWAY: Das Internet ist die neueste Möglichkeit zur Jobsuche. In den USA seit Jahren erfolgreich, bieten jetzt auch deutsche Anbieter interessante WEGE, die passende Stelle zu finden - ganz OHNE SCHLAGLÖCHER. ... Bei allen Online-Jobbörsen kann man auch selbst ein Stellengesuch aufgeben. Und gelangt so vielleicht auf ALTERNATIVROUTEN zum Ziel."

In dem ebenfalls der Zeitschrift freundin entnommenen Text "Das Geheimnis unseres Immunsystems" finden wir folgendes vor: "Eine starke TRUPPE: Ständig sind die ABWEHRZELLEN im Körper unterwegs, schwimmen durch das Lymphsystem auf der Suche nach FEINDEN... Für fast jeden EINDRINGLING gibt es eine SPEZIALEINHEIT, die sich sofort auf ihn STÜRZT. An VORDERSTER FRONT stehen die Freßzellen ... Die 'GENERÄLE' des ABWEHRSYSTEMS sind die T-Zellen: Sie IDENTIFIZIEREN unbekannte FEINDE, ORGANISIEREN über chemische Botenstoffe die ABWEHR und ZERSTÖREN auch selbst ERREGER. ... Aber die ABWEHRKRÄFTE sind nicht bei allen gleich STARK. Während sie bei 
manchen Menschen so gut wie jeden Bakterien- oder VirenANGRIFF problemlos ZURÜCKSCHLAGEN, müssen sie bei anderen schon vor minimalen ErregerINVASIONEN KAPITULIEREN... Ernährung. Ohne BAUMATERIAL, TREIBSTOFF und TRANSPORTMÖGLICHKEITEN ist auch die STÄRKSTE ARMEE hilflos." (26/96/S.55)

In diesen drei Fällen geht es darum, daß Wissensmuster (vgl. Sandig 1989: 134) aus einem Bereich der menschlichen Tätigkeit, der bekannt und vertraut ist, auf andere Gebiete übertragen werden: Angenehmer Komfort eines Appartements wird auf das Auto angewendet, das Wissensframe (vgl. auch sog. globale Muster bei Heinemann/ Viehweger 1991: 68 ff.) aus dem Bereich Verkehrswesen (Straßennetz und Bewegungsschnelligkeit auf Autobahnen) auf Wege und Umwege, wie man zu einer neuen Stelle kommen kann, und im dritten Fall unser allgemeines Wissen von den militärischen Organisationsstrukturen auf unser körperliches Abwehrsystem und seine Aktivität im Fall einer Krankheit. ${ }^{1}$

In allen drei Fällen haben wir somit ein Tertium comparationis als die Bedingung dafür, daß man bei zwei Bezeichnungsobjekten (Schippan 1984: 93), zwischen denen Ähnlichkeitsrelationen vorhanden sind, von metaphorischer Übertragung sprechen darf.

Was dabei unsere Aufmerksamkeit weckt, sind folgende zwei Fakten:

a) es geht um keine Einzelmetaphern auf der Lexemebene - wie z. B. spitze Bemerkung: eine solche, die wie spitze Gegenstände verletzen kann - sondern, wie oben bereits angedeutet, um komplexe, entfaltete, "umfassende" (a.a.O. : 211) Metaphern, die sich über den ganzen Text oder mindestens über größere Textsegmente hinweg erstrecken, und

b) diese komplexe Metaphorik ist nicht nur in literarischen Texten vorzufinden, sondern auch in Sach-, d. h. Gebrauchstexten.

Diesem Phänomen möchte ich in meinem Beitrag nachgehen und stelle mir dabei in Anbetracht der oben hypostasierten Problemstellung folgende Fragen:

1. Wie ist es um die komplexe Metaphorik auf der Textebene bestellt? Kann man in diesem Fall schlechthin von einer besonderen Textsorte sprechen, etwa von der Parabel, Allegorie, vom Gleichnis?

2. Welche Funktionen kommen der komplexen Textmetaphorik in Sachtexten zu?

3. Welche interkulturellen und intertextuellen Implikationen lassen sich dabei nachweisen?

B. Um diese Fragen beantworten zu können, versuche ich den unten angeführten Text aus der Zeitschrift Elle, der ein Gebrauchstext ist, zu analysieren. Für Gebrauchstexte ist es bekanntlich charakteristisch, daß sie einem mehr oder weniger

1 Sandig spricht in solchen Fällen von der "Militärmetaphorik" (1989: 134). 
praktischen Zweck im alltäglichen Leben dienen, z. B. dem Informieren, Berichten, Instruieren u. d. m. Nach Dimter (1981: 35) werden unter Gebrauchstexten jene Texte verstanden, mit denen in der Regel "kein besonderer ästhetisch-literarischer Anspruch" verbunden wird. Rolf sieht das Typische der Gebrauchstexte darin, daß in ihnen "mit der Realisierung einer Gebrauchstextsorte ein praktischer Zweck, ein in der Praxis auftauchendes Problem angegangen wird" (1993: 314), weshalb sie nicht für ein Publikum hergestellt werden und sich dadurch grundsätzlich von literarischen Texten unterscheiden, die in erster Linie dem ästhetischen Vergnügen frönen und künstlerische Ziele verfolgen (vgl. Wilpert 1969: 324). Daß Grenzen zwischen ihnen manchmal schwer zu ziehen sind, gilt auch als unbestritten. (Vgl. Brinker a.a.O.) Rolf hat in seiner Monographie zu Gebrauchstexten (1993: 165) über 2000 verschiedene Gebrauchstextsortenbezeichnungen herausgearbeitet, die lexikalisiert sind und zu denen u. a. auch Textsorten gehören wie z. B. Fahrplan des Zuges, Hausordnung, Rechtsmittelbelehrung, Vermißtenanzeige, Speisekarte, Korrekturvorschriften u. a. m. Der Rolfschen Klassifikation liegen die fünf Klassen der Sprechakte nach Austin und Searle zugrunde, so daß er assertive, direktive, kommissive, expressive und deklarative Gebrauchstextsorten unterscheidet (a.a.O.: 172). Das Basiskriterium ist somit die kommunikative Intention, die Illokution und die damit zusammenhängende Funktion einer jeweiligen Textsorte (a.a.O.: 311).

\section{Der Text:}

\section{Was uns stark macht}

Die richtige Einstellung und intelligente Strategien - das gibt Power für den Alltag. Elle sagt, was Goliath-Frauen falsch machen (viel Energieverlust, wenig Effekt). Und wie Sie eine David-Frau (gezielter Einsatz, perfekter output) werden

(I.) Die Puderdose liegt auf ihrem Schoß, der Schminkbeutel auf der Ablage rechts. Warum machen diese Deppen auf der Fahrerseite keinen Spiegel auf die Sonnenblende, flucht sie in sich hinein. Erste Ampel: Lippenkonturen, Lippenstift. Zweite Ampel: Kajal hinmalen. Kurz nach Ampel drei passiert es: Sie tritt so fest auf das Bremspedal, wie sie kann. Zu spät. Hastiger Griff zum Handy. "Ich komme leider erst um zehn, weil ich einen kleinen Auffahrunfall hatte." Beinahe dankbar ist sie für den Blechschaden: Zu spät gekommen wäre sie nämlich sowieso zu der seit langem angesetzten großen Konferenz, nur eben ohne triftige Entschuldigung.

(II.) Die Kollegin im Büro, der sie die Unfallursache gesteht, meint nur: "Sei froh, daß du noch beide Augen hast. Warum schminkst du dich auch beim Fahren?" Und sie antwortet in klassischer Selbstverteidigung: "War eben wieder dieser verdammte Streß."

(III.) Nur: Woher kam der? Die Kollegin hat zwei Kinder (wovon immer mindestens eines eine Kinderkrankheit durchmacht), einen Ehemann, einen kranken Vater im Stock darüber, und für ihren Weg zum Büro braucht sie eine halbe Stunde länger. Ihr Job ist mindestens so anstrengend wie der der anderen, und außerdem ist sie 
eine hingebungsvolle Gastgeberin mit einem kunterbunten großen Freundeskreis. Trotzdem wirkt diese Frau eigentlich nie gestreßt oder genervt. Sie dagegen hat einen betuchten Freund, keine Kinder, ein pflegeleichtes Appartement, und zweimal die Woche kommt eine Putzfrau. Trotzdem ist sie ständig im Streß oder einfach fertig mit den Nerven.

(IV.) Immer mehr Frauen kriegen einen Herzinfarkt, denn immer mehr Frauen leiden unter Streß. So triumphierten eine Zeitlang alle, die es schon immer gewußt hatten: Gratis gibt's die Emanzipation nicht. Und diese Superfrauen, die Kindererziehung, Karriere und Sex mit dem Partner gleichermaßen bravourös durchziehen, wären sowieso für den Infarkt prädestiniert. So paßt es ins Programm der Neider. Wahr ist es deshalb noch lange nicht.

(V.) Diejenigen, die beim Psychotherapeuten liegen und drei Tage krankgeschrieben werden wollen wegen vegetativer Dystonie, die Magen- und Hautprobleme haben, Spannungskopfschmerzen und alles andere, was unter "psychosomatisch" läuft, haben meistens nicht zuviel zu tun, sondern zuwenig Strategie. Wer wissen will, wie die richtige Strategie aussieht, möge nicht zum Telefonbuch greifen, um einen Kurs zu buchen bei den derzeit modischen Zeitplanunternehmen. Sondern zur Bibel. Dort findet sich im Alten Testament im 1. Buch Samuel die Geschichte von David (schön, aber schmächtig, unmuskulös und unerfahren) und Goliath (athletisch, erfahrener Schläger, Typ Bodybuilder). Im Schaukampf der beiden gewinnt bekanntermaßen der zarte David: Sein Sieg ist ein Sieg des Hirns über den Bizeps, der Intelligenz über die Potenz. Der Hirtenbub David setzt den Bodybuilder Goliath außer Gefecht, indem er mit einer Steinschleuder auf dessen Stirn zielt. Und trifft.

(VI.) Was David auszeichnet, zeichnet auch jene Frauen aus, die es schaffen, beruflich erfolgreich und privat glücklich, gutaussehend und dabei auch noch gutgelaunt zu sein. Die David-Tugenden.

1. David ist offen und beweglich. Er ist nicht bewehrt wie Goliath, der einen bleischweren Schuppenpanzer, Beinschienen und einen Helm aus Erz trägt. David tritt zum Kampf im Hemd an. Das heißt: David-Frauen wappnen sich nicht ängstlich mit Argumenten. Denn wer gnadenlos und perfekt gerüstet in einen Kampf geht - ob das eine Konferenz, eine private Diskussion oder ein Vorstellungsgespräch ist - hat es schwer. Ist zu sehr mit dem Ballast beschäftigt und nicht imstande, schnell und wendig die Richtung $\mathrm{zu}$ ändern. Wer unbeschwert und unbelastet reingeht, kann schnell, spontan und flexibel auf Unerwartetes reagieren. Die David-Frau sagt sich: Verpennt? Gut, dann geh ich eben ungeschminkt in die Konferenz. Die Goliath-Frau baut statt dessen den Auffahrunfall.

2. David läßt sich nicht bluffen. Goliath plustert sich vor dem Kampf fürchterlich auf, was alle verschreckt, nur David nicht. Er vermutet zu Recht hinter Goliaths Gerede eine unsichere Persönlichkeit. Das heißt: David-Frauen lassen sich von Verbalprotzen nicht einschüchtern. Sie nehmen instinktsicher wahr, wo und wie der andere zu verunsichern ist. Und sparen sich die Kraft, auf das Selbstlob von anderen einzugehen 
(genausowenig, wie sie von sich selbst behaupten müssen, sensationell zu sein). Goliath-Frauen verschleißen viel Energie und Phantasie, um mehr oder weniger wahrheitsgemäß zu berichten, wer sie alles unwiderstehlich, blitzgescheit etc. gefunden habe.

3. David ist sich sicher, daß er für die richtige Sache kämpft. Er glaubt daran, daß sein Ziel und sein ethischer Rückhalt richtig sind. Bei David-Frauen ist das ähnlich. Sie haben ein inneres Selbstverständnis, über das sie allerdings nicht ständig reden müssen. Daher sparen sie Zeit und Kraft, nach Motiven und Rechtfertigungen zu suchen für das, was sie tun oder lassen. Sie sagen sich beispielsweise: Meine Familie ist mir einfach am nächsten. Und haben dabei keine Gewissensbisse. Bei Goliath-Frauen haben Aktionen häufig nur ein Ziel: andere zu beeindrucken. Und sie neigen wie ihr Namenspatron dazu, sobald sie nervös werden, den Gegner zu beleidigen oder schlechtzumachen.

4. David ist ein geborener Stimmungsaufheller - obwohl er sieben Brüder hat, die nicht gerade nett sind zu ihm. Im Kapitel vor dem einschlägig bekannten wird erzählt, daß David den depressiven und daher oft bösartigen König Saul in gute Laune versetzte, indem er ihm auf der Zither vorspielte. David-Frauen beherrschen das ebenfalls: Sie lächeln, streicheln oder blödeln Widerstände einfach weg. Kaum legt so eine Frau der ergrimmten Kollegin oder dem streitbaren Gatten die Hand auf den Arm, werden die mild wie Sahnejoghurt. Goliath-Fauen schaffen das nicht: Sie tun sich schwer, über den eigenen Schatten zu springen, denn es ist ihnen wichtiger, recht zu haben als Frieden zu finden.

5. David beherrscht die Techniken, die für ihn wichtig und ihm nützlich sind. Und die ihm liegen. Er kann eines perfekt: mit der Steinschleuder umgehen. Die angebotene Rüstung des Königs probiert er zwar brav an, zieht sie aber gleich wieder aus. Das ist nicht sein Ding. David-Frauen wissen auch genau, was sie beherrschen, wo sie treffsicher sind. Sie wollen nicht alles können, konzentrieren sich auf ihr besonderes Talent und haben deswegen Erfolg. Goliath-Frauen wollen überall die besten sein (schon eine Freundin, die fünf Kilo weniger auf den Knochen hat, ist ihnen ein Dorn im Auge). Das streßt natürlich und entmutigt sie schließlich.

6. David ist ein Einzelkämpfer. Goliath tritt mit Schildträgern auf, Daviđ hingegen, das schmale Hemd, ohne jeden Geleitschutz. Und daher entscheidet er auch schnell und allein, nachdem der Riese bewußtlos am Boden liegt: Jetzt heißt es hingehen und den Kerl köpfen. David-Frauen sind trotz Familie oder anderer Bindungen Einzelkämpferinnen. Was allerdings nicht heißt, daß sie nicht teamfähig sind. Aber sie wissen, daß es bestimmte Entscheidungen gibt, die sie alleine fällen müssen, ohne vorher eine Meinungsumfrage zu starten. Goliath-Frauen geben sich zwar selbst- und siegessicher. Weil sie aber im Grunde unsicher sind, holen sie vor einer Entscheidung so viele Meinungen ein, daß sie schließlich nicht mehr wissen, was ihre eigene war oder ist. Was sie natürlich nie zugeben würden: Sie machen das auch, um einen Schuldigen zu haben, falls sich die Entscheidung als falsch entpuppt. 
7. David kann seine Kräfte einschätzen, seine Schwächen zugeben. Und ist sich immer dessen bewußt, daß es etwas Größeres gibt auf dieser Welt als ihn. "Der Herr führt den Kampf", erklärt er den Gegnern. Im Fall Davids ist es sein Glaube, der ihn angstfrei macht. Er kann seine eigene Bedeutung relativieren. Anders gesagt: Er nimmt sich nicht zu wichtig. David-Frauen überschätzen sich und ihr Einzelschicksal genausowenig. Daher hadern sie auch nicht mit ihrem Schicksal. Goliath-Frauen fühlen sich dauernd vom Unglück verfolgt, weil sie in ihrer Nabelschau das Leid der anderen nicht wahrnehmen. Und weil sie nichts kennen, das ihnen wichtiger wäre als ihr eigenes Wohlergehen.

(VII.) Wenn eine Goliath-Frau zur David-Frau werden will, muß sie damit beginnen, sich selber aus dem Mittelpunkt zu rücken. Sie muß lernen zuzugeben, daß ihr Streß selbstgemacht ist. Und sie muß es schaffen, über ihre Streß-Reaktionen zu lachen. Was sich übrigens trainieren läßt: Wer sich beim Ausrasten und entnervten Rumfuhrwerken einmal zuschaut wie bei einem Film, muß das Ganze zwangsläufig saukomisch finden. Die Folge ist ebenso einleuchtend wie hilfreich: Wer sich selber auf den Arm nehmen kann, nimmt manches andere auch viel leichter.

EVA GESINE BAUR Elle 12 (Dezember)/96, S. 78-84.

Die Proposition unseres zu analysierenden Mustertextes ist die gegenwärtige aktuelle Problematik der Frauen, ihre Überlastung und der damit zusammenhängende Streß, mit dem sie meistens nicht fertig werden, wenn sie Beruf und Familie unter einen Hut bringen wollen. Es wird aber auch ein Ausweg aus dieser schwierigen Lage suggeriert, und zwar in der richtigen Strategie, die den Frauen in den alltäglichen Auseinandersetzungen mit anfallenden Verpflichtungen meistens fehlt.

Nach Rolf ginge es in unserem Fall um einen z. T. assertiven Text (subsidiäre Funktion), denn "die assertiven Textsorten dienen der Informationsvermittlung" (1993: 172), hauptsächlich aber um eine direktive Textsorte (dominierende Funktion), u. zw. um einen "nicht-bindenden" direktiven Text "bei Textrezipienteninteresse" (a.a.O.: 257), denn darin "richtet sich das Interesse des Textrezipienten auf ein Problem" (ibid.), das vom Adressaten nach Möglichkeit bewältigt werden soll (vgl. a.a.O.: 223 u. 245). Mit anderen Worten haben wir es in diesem Fall mit einem psychologischen Beratungstext oder mit Franke (1996: 249 ff.) mit einem ratgebenden Aufklärungstext zu tun.

Welche Strategien sich Frauen aneignen sollten, damit sie Herr der Lage werden, wird vermittelt, und zwar so, daß in Gedanken beim bekannten David-GoliathZweikampf aus der Bibel zugesehen wird. ${ }^{2}$ Diese Bibelgeschichte wird als Folie dem gesamten Gebrauchstext unterlegt, sie dient als komplexe Metapher, auf der die Autorin unseres aufklärenden Gebrauchstextes Schritt für Schritt erklärt, was eine Goliath-Frau enzyklopädische Wissen der Verfasserin hindeutet. 
- eine solche ohne Strategie - zu tun hat, um eine David-Frau zu werden - eine solche, die - mit richtiger Strategie gewappnet - erfolgreich den Alltag meistert. ${ }^{3}$

Der Reihe nach werden jene Tugenden Davids geschildert, die ihn, obwohl schmächtig und unmuskulös, dazu befähigt haben, den athletischen Schläger, Typ Bodybuilder, Goliath, zu besiegen.

1. Im VI. Absatz (1) lesen wir: "David ist offen und beweglich. Er ist nicht bewehrt wie Goliath..." Das ist jenes Tertium comparationis, auf dem eindeutig und explizit eine Parallele zu David-Frauen gezogen wird: "Das heißt: David-Frauen wappnen sich nicht ängstlich mit Argumenten." Dieses Tertium comparationis - Offenheit und Beweglichkeit -ist der Bibel entnommen, es ist eine Tatsache, von der ausgegangen wird, eine thematische Problemstellung (vgl. Bračič 1994: 15), die analysiert und erklärt wird. Hier drängt sich der Begriff des Explanandums von Brinker auf, wenn er von der explikativen thematischen Entfaltung (1988: 64 ff.) spricht. Diese verläuft in der von mir analysierten Textpassage jedenfalls nicht ganz linear und durchsichtig: während äußere Umstände - nach Brinker (1988) sog. Anfangs- oder Randbedingungen (65) ("Er ist nicht bewehrt ..., ... tritt zum Kampf im Hemd an") - noch aus der biblischen Sphäre des Zweikampfes stammen, so greift die allgemeine Gesetzmäßigkeit der erklärenden Kategorie, nach Brinker (a.a.O.) eine Komponente des Explanans, z. T. schon auf die Sphäre der David-Frau über: "... wer gnadenlos und perfekt gerüstet in einen Kampf geht - ob das eine Konferenz, eine private Diskussion oder ein Vorstellungsgespräch ist - hat es schwer. Ist zu sehr mit dem Ballast beschäftigt und nicht imstande, schnell und wendig die Richtung zu ändern." Aufgrund dieses Vergleichs wird auch das erwünschte Explanandum auf der Ebene der David-Frau herbeigeführt: /"Wer unbeschwert und unbelastet reingeht (z. B. in eine Konferenz, S.B.)/, kann schnell, spontan und flexibel auf Unerwartetes reagieren." Und diese David-Frau-Tugend wird noch zusätzlich exemplifiziert4: "Die David-Frau sagt sich: Verpennt? Gut, dann geh ich eben ungeschminkt in die Konferenz. Die Goliath-Frau baut statt dessen den Auffahrunfall" (vgl. Absatz I.).

In diesem einen Absatz begegnet eine kunterbunte, stark verschränkte Mischung verschiedener Elemente, die jedoch aufeinander abgestimmt sind und überzeugend wirken. Mit Barbara Sandig (1989: 136). könnte man von verschiedenen Mustermischungen sprechen: Das Wissensmuster der Bibel wird mit dem Wissensmuster der Alltagserfahrung der Frau gemischt, das Handlungsmuster des Beschreibens kombiniert sich mit Elementen der Handlungsmuster Vergleichen, Erklären und Explizieren. David steht Goliath gegenüber, David-Frau der Goliath-Frau, was David Goliath überlegen macht, wird auf Frauen übertragen und angewendet. Das Ergebnis dieser Mustermischung ist ein effektvolles metaphorisches Bild. Die zu vermittelnden Sachverhaltszusammenhänge werden also nicht direkt versprachlicht. Eine

3 Im folgenden metaphorischen Textgestaltungsprozeß sehe ich eine Komponente der Textlokution. Auf die Begründung dafür werde ich im weiteren noch zurückkommen. (S. Anm. 5.)

4 Vgl. zur Frage der Exemplifizierung bei Greule 1995: 64. 
unmittelbare Darbietungsform würde nämlich der Überzeugung von Medienpraktikern und -wissenschaftlern zuwiderlaufen, daß Ratschläge und Informationen in Ratgebertexten auf anschauliche und unterhaltsame Weise darzubieten seien, um die anonyme Monotonie und Unidirektionalität der massenmedialen Kommunikation zu überspielen (vgl. Franke 1996: 258). Mit der Metaphorisierung wird über Assoziationsbeziehungen in die Darbietungsform eine neue Sichtweise, eine Perspektivierung gebracht, denn mit Weinrich werden "Analogien ... der Natur nicht abgelesen, sondern in sie hineingelesen als unsere Entwürfe, als unsere Hypothesen" (zit . nach Lewandowski 1994: 708).

Diese Metaphorik zieht sich praktisch durch den ganzen restlichen Text hindurch. Auf ähnliche Weise werden so auch weitere Tugenden Davids erklärt und auf die David-Frau übertragen. In gedrängter Form führe ich hier die weiteren David-Tugenden, wie sie im Text geschildert sind, an:

- Wie sich David nicht von Goliath bluffen ließ (Absatz VI./2.), weil er hinter Goliaths Gerede zu Recht eine unsichere Persönlichkeit vermutete, so lassen sich auch David-Frauen nicht von Verbalprotzen einschüchtern.

- David ist sich sicher, daß er für die richtige Sache kämpft (Absatz VI./3.) und einen ethischen Rückhalt hat, auch David-Frauen haben ein inneres Selbstverständnis, aus dem sie Kraft schöpfen.

- David ist ein Stimmungsaufheller (Absatz VI./4.), auch David-Frauen lächeln, streicheln oder blödeln Widerstände einfach weg.

- David probiert zwar die angebotene Rüstung des Königs brav an (Absatz VI./5.), zieht sie aber gleich wieder aus und bleibt bei dem, was er beherrscht, bei der Steinschleuder, mit der er dann Goliath auch besiegt - auch David-Frauen wollen nicht alles können und konzentrieren sich auf ihr besonderes Talent.

- Sowie David ein Einzelkämpfer ist (Absatz VI./6.) und somit genau weiß, wann Goliath zu köpfen ist, genauso fällt die zwar durchaus teamfähige David-Frau selbst wichtige Entscheidungen und wälzt die Verantwortung nicht auf andere ab, wenn in der Folge was schief geht.

- David kann seine Kräfte einschätzen und seine Schwächen zugeben. Auch David-Frauen beherrschen die Kunst des Relativierens (Absatz VI./7.) und überschätzen sich und ihr Schicksal nicht.

$\mathrm{Da}$ gerade das Gegenteil jeweils für Goliath bzw. Goliath-Frauen zutrifft, muß nicht extra hervorgehoben werden.

C. Aufgrund des bisher Ausgeführten erhebt sich erneut die eingangs bereits gestellte Frage: Haben wir in unserem Fall neben der oben erwähnten Wissensmusterund Handlungsmustermischung auch eine Textsortenmischung? Ist unser Text ein Gleichnis, ist er eine Parabel, eine Allegorie oder doch nur ein aufklärender Sachtext? 
Bevor diese Frage beantwortet werden kann, müßten wir uns einer anderen Problemstellung zuwenden. Bekanntermaßen sind Allegorie, Gleichnis und Parabel literarische Textsorten, die, häufig auf biblische Ereignisse züruckgreifend, eine Lehre vermitteln. Allegorie wird dabei als Symbolisierung nicht unbedingt auf der Textebene realisiert, ein Gleichnis beruht auf direkten Verknüpfungen und Parallelen im Sinne von "so ...wie" (Meyers Lexikon 1981: 10/455), während Parabeln unter Berufung auf andere Geschichten mehr indirekt zu belehren suchen. Propositions- und illokutionsmäßig kämen also für unsere Zwecke in Frage Gleichnis und Parabel: beide beziehen sich referentiell auf eine Geschichte, aus der eine Lehre zu ziehen ist.

Bezüglich der Textlokution, d. h. der sprachlich-formulativen Mittel, die bei solchen Analysen als dritter zu beachtender Parameter heranzgezogen werden (vgl. bei Fix 1993: 122), möchte ich zwei weitere Aspekte unter die Lupe nehmen: die Textstruktur und die Textsyntax. ${ }^{5}$

Textstrukturell scheint es im Text einen zentralen Punkt, eine Art Achse zu geben, um die sich der Text dreht. Dieser zentrale Punkt ist repräsentiert durch die Äußerung im V. Absatz: "Diejenigen, die beim Psychotherapeuten liegen und drei Tage krankgeschrieben werden wollen wegen vegetativer Dystonie, die Magen- und Hautprobleme haben, Spannungskopfschmerzen und alles andere, was unter 'psychosomatisch' läuft, haben meistens nicht zuviel zu tun, sondern zuwenig Strategie." Das ist eine von der Textautorin aufgestellte These, die durch den Modaloperator meistens in ihrer Extension relativiert wird. Tatsachenfeststellungen, auf die sich diese These stützt sind (IV. Absatz): "Immer mehr Frauen kriegen einen Herzinfarkt, denn ..." Im letzten (VII.) Absatz befindet sich die Regel, die besagt, weshalb die These plausibel ist: "Wenn eine Goliath-Frau zur David-Frau werden will, muß sie damit beginnen, sich selber aus dem Mittelpunkt zu rücken. Sie muß lernen ..." Das in den ersten drei Absätzen enthaltene Textsegment ist eine Art Einbettung, die die These und Tatsachenfeststellungen situiert. Diese Untergliederung stimmt mit den Kategorien der argumentativen thematischen Entfaltung nach Brinker (a.a.O.: $68 \mathrm{ff}$.) überein, u. zw. mit den Parametern Einbettung, These, Argument, Schlußregel, Modaloperator. Es fehlt eigentlich die sog. Stützung mit der Wertbasis, und diese Stützung ist eben das mittlere Textsegment (ab Zeile 4 des V. Absatzes), wo auf die oben bereits dargelegte Art und Weise explizit erläutert wird, was David und David-Frauen einerseits bzw. Goliath und Goliath-Frauen andererseits gemeinsam haben. Unter Berufung auf die Bibel, auf die christliche Tradition als Fundament der

5 Zur Textlokution gehören $\mathrm{m}$. E. verschiedene oben dargelegte Mustermischungen, aber auch der Einsatz von Sprachhandlungstypen, aus der Überlegung heraus, daß bei der durchaus berechtigten Transposition der Sprechakttheorie von Satz auf Text, der Text als Träger ein und derselben Handlung verschiedene Illokutionen realisieren kann. Als Beispiel sei die Erzählung mit den Intentionen des Unterhaltens oder Werbens angeführt. Sprachhandlungstypen können m. E. nicht als Textillokutionen gelten, sie sind vielmehr das Mittel, der Weg, der zum Ziel, d. h. zu einer Textillokution führt. Ansonsten geraten wir bei jeder Textmustermischung als indirektem Sprechakt in eine Aporie: Erzählen wird etwa in Werbetexten eingesetzt, um zu werben, und nicht, um zu erzählen. (So auch bei Fix 1993: 124.) 
deutschen Kultur (Schlosser 1983: 251) wird eine Art Stützung, eine moralische Kraft und Berechtigung für das Argumentieren gesichert.

Was die Textsyntax anbelangt, läßt sich folgendes vermerken:

Die Textsyntax zeichnet sich hier dadurch aus, daß sie klar und explizit ist: keine Komprimierungen und Verdichtungen sind vorhanden, die häufig in poetischen Texten vorkommen und deren Auflösungen zwangsläufig zu einseitigen Festlegungen führen (vgl. Fix 1997: 11). Die Textkohäsion ist evident, es gibt keine Syntax "in den Köpfen" der Leser (a.a.O.: 1), deren Rekonstruktion auf dem Papier mit einer Bedeutungsvagheit verschiedener Lesarten verbunden ist. Zur Bekräftigung dieser Feststellungen führe ich für den mittleren Teil des Textes (Kapitel VI./1.-7.) zwei Beweise auf:

1. Eindeutige Vergleichskonnektoren: daß heißt (in VI./1. und VI./2.):"Das heißt: David-Frauen wappnen sich nicht ängstlich mit Argumenten"; ähnlich (in VI./3.): "Bei David-Frauen ist das ähnlich"; wie (Absatz VI./3.): "Sie neigen wie ihr Namenspatron dazu ..."; ebenfalls (VI./4.): "David-Frauen beherrschen das ebenfalls ..."; auch (VI./5.): "David-Frauen wissen auch ..."; genausowenig (VI./7.): "David-Frauen überschätzen sich ... genausowenig."

2. Präzise Erklärungen des Tertium comparationis und dessen Anwendung auf Frauenverhältnisse (z. B. in VI./2. oder in VI./5.): Die Metaphorik wird auseinandergenommen, es geht beinahe um eine Vivisektion des metaphorischen Mechanismus bis ins Eingeweide hinein.

Wozu? Wir müssen uns vor Augen führen, daß es sich in unserem Fall um einen aufklärenden Gebrauchstext handelt. Und die Devise dieses aufklärenden Gebrauchstextes könnte heißen: Sprachliche Bilder ja, aber keine Zweideutigkeiten. Die grundlegende Intention eines aufklärenden Sachtextes liegt (nach Franke 1996) darin, daß das vorhandene Wissensdefizit bei einem dispersen Publikum behoben wird (257). Das deckt sich mit der Erwartung des potentiellen Leserkreises und hängt langfristig mit der redaktionellen Politik einer Zeitschrift zusammen.

Vor diesem Hintergrund läßt sich auch der wesentliche Unterschied zwischen der sprachlichen Bildlichkeit in Gebrauchstexten und in literarischen Texten erörtern. In literarischen Texten scheint alles Übertragene, so auch die Metaphorik, im Dienst eines ästhetischen/künstlerischen Genusses zu stehen, Doppelbödigkeiten sind erwünscht, der Rezipient sieht mit voller Akzeptanz seine Rolle darin, sich aufgrund verschiedener Inferenzbeziehungen mit uneigentlichen Versprachlichungen auseinanderzusetzen, um unterschiedliche Lesarten herauszufinden. Das Bildliche in Sachtexten dagegen ist wenn auch bisweilen auf kleinere Rezipientengruppen beschränkt - eindeutig und auf keine Verfremdungseffekte bedacht, hauptsächlich dient die Bildlichkeit in Sachtexten der Argumentation und Verlebendigung, Ästhetisches und Unterhaltung können dabei nur als nebensächliche Begleiterscheinungen betrachtet werden. So wird es plausibel, daß Mustermischungen auf der Textebene in erster Linie der Textfunktion dienen: Daraus ist zu schließen, daß in unserem Fall das in das mittlere Textsegment (Absatz VI./1.-7.) eingebaute Gleichnis - um die Differenzierung von der Parabel außer acht zu lassen - ein demontiertes, verzerrtes ist, weil die prototypische Gleichnislokution mit 
ihrer Verschlüsseltheit ein zu hohes Risiko für die Akzeptabilität seitens des Rezipienten in sich birgt. $^{6}$

D. Die beiden eingangs gestellten Fragen scheinen mir damit beantwortet zu sein. Es bleibt noch die dritte bezüglich der Interkulturalität und Intertextualität in Sachtexten.

Hierbei scheint interessant $\mathrm{zu}$ sein, daß die Bibel nicht nur in unzähligen literarischen Werken intertextuell überarbeitet wird, ${ }^{7}$ sondern daß auf sie nach 2000 Jahren auch noch so aktuell referiert werden kann, wie in unserem Beispieltext. Die Bibel gehört mit der antiken Mythologie und der altorientalischen Philosophie (man denke nur an den Westöstlichen Divan bei Goethe; orientalische Motivik finden wir bekanntlich auch bei Brecht) zu jenen Schätzen menschlichen Schaffens, die nicht nur interkulturell von Bedeutung sind, sondern die über alle Grenzen hinweg mit einem geradezu transkulturellen Gepräge in allen menschlichen Tätigkeitsbereichen, allen Textsorten, Gattungen und Stilen direkt oder indirekt ihren Niederschlag finden.

Ich möchte diesen meinen Beitrag mit einer unwissenschaftlichen Bemerkung abschließen. In unserem Text geht es um einen Topos, der womöglich vor allem bei kleineren Nationen - wie Juden, aber auch Slowenen - in vielen Variationen bekannt ist: Dem Schwachen, Verschmähten wird plötzlich wie durch ein Wunder eine Kraft zuteil, mit der er den bösen Riesen besiegt. Wir Slowenen kennen z. B. in unserer Literatur die Geschichten von Martin Krpan oder von Peter Klepec. Die Tatsache, daß der Name David bei uns besonders bei der jüngeren Generation sehr verbreitet ist, deutet auf einen vielleicht mehr unterbewußten Sinn des Volkes für die Metaphorik hin. Es steht außer Zweifel, daß die oben dargebotenen Empfehlungen, wie ein GoliathMenschenschlag sich David-Tugenden aneignet, auch für die männliche Welt Geltung haben können.

\section{Bibliographie:}

Bračič, Stojan (1994): Statische und dynamische Komponenten der Textkonstitution. In: Halwachs, Dieter W./Stütz, Irmgard (Hrsg.): Sprache - Sprechen - Handeln, Akten des 28. Linguistischen Kolloqiums, Graz 1993. Band 2, S. 15-20.

6 Zu verweisen wäre dabei auf Kallmeyers Ansichten bei der polyfunktionalen Vertextung in einer komplexen Isotopie: Während auf komische Effekte bedachte polyfunktionale Vertextungen im Analyseverfahren monosemiert werden müssen (etwa in Witzen), "findet in ästhetischen Texten eine solche Reduzierung nicht statt. Diese sind gerade durch ihre semantische Offenheit charakterisiert" (Kallmeyer et al. 1980: 159).

7 Ich verweise hier lediglich auf Dietrich (1995: 252), der die biblische David-Goliath-Geschichte bei vier modernen Autoren (Stefan Heym, Joseph Heller, Torgny Lindgren und Grete Weil) verfolgt, sowie auf Meyers Enzyklopädisches Lexikon (1980 : Bd.6: 309), dem zufolge das Schicksal Davids in zahlreichen Bearbeitungen dichterisch gestaltet wurde, wobei zu den bevorzugten Themen eben auch der Kampf mit dem Riesen Goliath zählte. 
Brinker, Klaus (19882): Linguistische Textanalyse. Eine Einführung in Grundbegriffe und Methoden. Berlin: Schmidt. (Grundlagen der Germanistik 29)

Dietrich, Walter (1995): Der Fall des Riesen Goliat: Biblische und nachbiblische Erzählversuche. In: Ebach, Jürgen/Faber, Richard (Hrsg.): Bibel und Literatur. München: Fink. S. 241-258.

Dimter, Matthias (1981): Textklassenkonzepte heutiger Alltagssprache. Kommunikationssituation, Textfunktion und Textinhalt als Kategorien alltagssprachlicher Textklassifikation. Tübingen: Niemeyer. (Reihe Germanistische Linguistik 32)

Fix, Ulla (1993): Die "Gattung Grimm", Andersens Märchen "Das häßliche junge Entlein" und das "Märchen vom häßlichen Dieselein". Ein Textmustervergleich. In: Wellmann, Hans (Hrsg.): Grammatik, Wortschatz und Bauformen der Poesie in der stilistischen Analyse ausgewählter Texte. Heidelberg: C. Winter. (Sprache Literatur und Geschichte 10) S. 113 - 128.

Fix, Ulla (1996): Was ist aus André Jolles 'Einfachen Formen' heute geworden? Eine kulturanalytische und textlinguistische Betrachtung. In: Hertel, Volker/Barz, Irmhild/ Metzler, Regine/Uhlig, Brigitte (Hrsg.): Sprache und Kommunikation im Kulturkontext, Beiträge zum Ehrenkolloquium aus Anlaß des 60. Geburtstages von Gotthard Lerchner. S. 105-120.

Fix, Ulla (1997): Die Wörter auf dem Papier und die Grammatik in den Köpfen. Zur Textualität und zu Lesarten von "grammatikarmen" Texten. Unveröffentlicht.

Franke, Wilhelm (1996): Ratgebende Aufklärungstexte. Überlegungen zur linguistischen Beschreibung ratgebender Beiträge der Massenmedien. In: ZGL 24, S. 249-272.

Greule, Albrecht (1995): Möglichkeiten und Grenzen der textgrammatischen Analyse. In: Studia Germanica Posnaniensia XXI, S. 55-65.

Heinemann, Wolfgang/Viehweger, Dieter (1991): Textlinguistik. Eine Einführung. Tübingen: Niemeyer. (Reihe Germanistische Linguistik 115)

Kallmeyer, Klein, Meyer-Hermann, Netzer, Siebert $\left(1980^{3}\right)$ : Lektürekolleg zur Textlinguistik. Band 1: Einführung. Königstein/Ts: Athenäum.

Lewandowski, Theodor (1994): Linguistisches Wörterbuch 2. Heidelberg, Wiesbaden: Quelle \& Meyer. (Uni-Taschenbücher 1518)

Meyers Enzyklopädisches Lexikon in 25 Bänden. Bd. 6 (1980), Bd. 10 (1981). Mannheim: Bibliographisches Institut.

Rolf, Eckard (1993): Die Funktionen der Gebrauchstextsorten. Berlin, New York: de Gruyter.

Sandig, Barbara (1989): Stilistische Mustermischungen in der Gebrauchssprache. In: Zeitschrift für Germanistik 2, S. 133-150.

Schippan, Thea (1984): Lexikologie der deutschen Gegenwartssprache. Leipzig: VEB: Bibliographisches Institut.

Schlosser, Horst Dieter (1983): dtv-Atlas zur deutschen Literatur. Tafeln und Texte. München: Deutscher Taschenbuch Verlag.

Wilpert, Gero von (19695): Sachwörterbuch der Literatur. Stuttgart: Kröner. 


\section{MEDKULTURNI IN MEDBESEDILNI ELEMENTI V NELITERARNIH BESEDILIH}

Metaforika se ne kaže samo na besedni, temveč v kompleksnejši obliki tudi na besedilni ravni. Vendar ne le v literarnih besedilih, temveč tudi v neumetniških besedilih vsakdanjega življenja. $\mathrm{V}$ literamih besedilih je metaforika sama sebi namen, je kot cilj komunikacije estetsko vprašanje. $V$ neliterarnih besedilih pa služi predvsem slikovitosti podajanja in povečanju pozornosti bralca/poslušalca in ne sme biti dvoumna, zlasti če gre za svetovalna besedila s sporočevalno intencijo, pomagati naslovniku iz zanj neugodnega položaja.

Mešanje besedilnih vzorcev pripelje $v$ literamih besedilih do novih podvzorcev, kot so alegorija, parabola, prilika. Takšno kombinacijo besedilnih vzorcev zasledimo tudi v neliterarnih besedilih, je pa $v$ njih zaradi zgoraj navedenih razlogov manj dosledna, saj se ravna po načelu: biti slikovit, a ne dvoumen.

Biblija ni le vir za kompleksno metaforiziranje v umetniških besedilih, ampak se njena medkulturna, če že ne kar nadkulturna potenca kaže tudi v tem, da se bolj ali manj neposredno sklicujejo nanjo tudi neliterarna besedila. 